\title{
Efeitos da Associação entre Pequenas Doses Subaracnóideas de Morfina e Cetoprofeno Venoso e Oral em Pacientes Submetidas à Cesariana *
}

\section{Effects of Low Spinal Morphine Doses Associated to Intravenous and Oral Ketoprofen in Patients Submitted to Cesarean Sections}

Eliana Marisa Ganem, TSA ${ }^{1}$; Norma Sueli Pinheiro Módolo, TSA ${ }^{1}$; Fábio Ferrari ${ }^{2}$; Francisco Carlos Obata Cordon ${ }^{2}$; Edgar Shiguero Koguti ${ }^{2}$; Yara Marcondes Machado Castiglia, TSA ${ }^{3}$

\section{RESUMO}

Ganem EM, Módolo NSP, Ferrari F, Cordon FCO, Koguti ES, Castiglia YMM - Efeitos da Associação entre Pequenas Doses Subaracnóideas de Morfina e Cetoprofeno Venoso e Oral em Pacientes Submetidas à Cesariana

JUSTIFICATIVA E OBJETIVOS: Pequenas doses subaracnóideas de morfina são eficazes em reduzir a dor pós-operatória de pacientes submetidas à cesariana, com menor incidência de efeitos colaterais. O objetivo desta pesquisa foi avaliar a qualidade da analgesia pós-operatória e a ocorrência de efeitos colaterais em pacientes submetidas a cesarianas, sob anestesia subaracnóidea com bupivacaína hiperbárica e morfina nas doses de 0,05 mg e 0,1 mg, associadas ao cetoprofeno pelas vias venosa e oral.

MÉTODO: Participaram do estudo 60 gestantes de termo, estado físico ASA I e II, que foram submetidas à cesariana eletiva. As pacientes foram divididas em dois grupos: grupo 1 morfina 0,1 mg, grupo 2 - 0,05 mg, associada a $15 \mathrm{mg} d e$ bupivacaína hiperbárica. Todas receberam cetoprofeno (100 mg) por via venosa no per-operatório e por via oral a cada 8 horas no primeiro dia de pós-operatório. As pacientes foram avaliadas 6, 12 e 24 horas após o término da cirurgia, com relação à intensidade da dor e presença de efeitos colaterais (sedação, prurido, náusea e vômito). A presença destes últimos também foi avaliada no per-operatório.

RESULTADOS: Ambos os grupos foram idênticos quanto aos dados antropométricos e à duração da cirurgia e da anestesia. Também foram homogêneos com relação à intensidade da dor pós-operatória e à presença de prurido, sedação, náusea e vômito.

CONCLUSÕES: A morfina, nas doses de 0,05 mg e 0,1 mg administradas no espaço subaracnóideo, associada ao

\footnotetext{
* Recebido do (Received from) CET/SBA do Departamento de Anestesiologia da Faculdade de Medicina de Botucatu (FMB - UNESP), Botucatu, SP

1. Professora Adjunta Livre Docente do CET/SBA do Departamento de Anestesiologia da FMB - UNESP

2. Ex-Residente do CET/SBA do Departamento de Anestesiologia da FMB - UNESP

3. Professora Titular do CET/SBA do Departamento de Anestesiologia da FMB - UNESP
}

\footnotetext{
Endereço para correspondência (Correspondence to)

Profa. Dra. Eliana Marisa Ganem

Dept ${ }^{\circ}$ de Anestesiologia da FMB - UNESP

Distrito de Rubião Junior

18618-970 Botucatu, SP
}

Apresentado (Submitted) em 07 de outubro de 2002

Aceito (Accepted) para publicação em 16 de dezembro de 2002

(C) Sociedade Brasileira de Anestesiologia, 2003 cetoprofeno pelas vias venosa e oral, apresentou a mesma qualidade de analgesia pós-operatória e determinou a mesma ocorrência de efeitos colaterais.

Unitermos: ANALGÉSICOS, Opióides: morfina, Antiinflamatório: cetoprofeno; CIRURGIA, Obstétrica: cesariana; TÉCNICAS ANESTÉSICAS, Regional: subaracnóidea

\section{SUMMARY}

Ganem EM, Módolo NSP, Ferrari F, Cordon FCO, Koguti ES, Castiglia YMM - Effects of Low Spinal Morphine Doses Associated to Intravenous and Oral Ketoprofen in Patients Submitted to Cesarean Sections

BACKGROUND AND OBJECTIVES: Low spinal morphine doses are effective in relieving postoperative pain of patients submitted to Cesarean sections, with low incidence of side-effects. This study aimed at evaluating postoperative analgesia and the incidence of side-effects in patients submitted to $\mathrm{Ce}$ sarean sections under spinal anesthesia with hyperbaric bupivacaine and $0.05 \mathrm{mg}$ and $0.1 \mathrm{mg}$ morphine associated to intravenous and oral ketoprofen.

METHODS: Sixty pregnant women, physical status ASA I and II, undergoing elective Cesarean sections, were divided in two groups: group 1 patients were given $0.1 \mathrm{mg}$ spinal morphine, while group 2 received $0.05 \mathrm{mg}$ morphine, both associated to 15 mg hyperbaric bupivacaine. All patients received perioperative $100 \mathrm{mg}$ intravenous ketoprofen and oral ketoprofen at 8-hour intervals in the first postoperative day. Patients were assessed at 6, 12 and 24 hours after surgery for pain intensity and side-effects (sedation, pruritus, nausea and vomiting). Side-effects were also evaluated in the perioperative period.

RESULTS: Both groups were similar in demographics and surgery and anesthesia duration. They were also homogeneous in postoperative pain intensity and presence of pruritus, sedation, nausea and vomiting.

CONCLUSIONS: Spinal $0.05 \mathrm{mg}$ and $0.1 \mathrm{mg}$ morphine associated to intravenous and oral ketoprofen have provided the same postoperative analgesia and have determined the same incidence of side-effects.

Key Words: ANALGESICS: Opioids: morphine, Anti-inflamatory: ketoprofen; ANESTHETIC TECHNIQUES: Regional: spinal block; SURGERY, Obstetric: cesarian section

\section{INTRODUÇÃO}

$\Lambda$ anestesia subaracnóidea é técnica muito utilizada para Arealização de cesarianas. É de fácil execução e de rápido início de ação, produzindo excelente relaxamento muscular e analgesia intensa, oferecendo condições per-operatórias ideais ${ }^{1,2}$. 
Desde o descobrimento de receptores opióides na medula espinhal na década de 70 a aplicação clínica de opióides, tanto no espaço peridural como no subaracnóideo, revolucionou a analgesia obstétrica ${ }^{3}$. O fentanil, o sufentanil e a morfina são os opióides que podem ser adicionados ao anestésicolocal com a finalidade de melhorar a analgesia no per-operatório ${ }^{4}$, ao passo que a morfina é a droga de escolha para analgesia no pós-operatório ${ }^{5}$.

Os opióides, contudo, desencadeiam alguns efeitos colaterais, como náusea, vômito, prurido, depressão respiratória, retenção urinária e sedação, cuja intensidade pode ser dependente da dose empregada ${ }^{6}$.

A dose de morfina recomendada para ser administrada no espaço subaracnóideo é de $0,1 \mathrm{mg}^{7,8}$, porém existem trabaIhos clínicos demonstrando que a morfina atingiria seu efeito máximo com doses inferiores a $0,1 \mathrm{mg}^{5}$. Acredita-se que doses menores de morfina pela via subaracnóidea associadas a outras drogas analgésicas possam ser eficazes em reduzir a dor pós-operatória com menor incidência de efeitos colaterais.

O objetivo desta pesquisa foi avaliar a qualidade da analgesia pós-operatória e a ocorrência de efeitos colaterais em pacientes submetidas à cesariana, sob anestesia subaracnóidea, com bupivacaína e morfina nas doses de 0,05 mg e 0,1 $\mathrm{mg}$, associadas ao cetoprofeno pelas vias venosa e oral.

\section{MÉTODO}

Após a aprovação do Comitê de Ética em Pesquisa Clínica e o consentimento por escrito das parturientes, participaram do estudo 60 gestantes de termo, estado físico ASAI, que foram submetidas à cesariana eletiva, com fetos de termo e sem sofrimento. As pacientes foram distribuídas, por sorteio, em dois grupos que se diferenciaram pela dose de morfina que foi adicionada ao anestésico local administrado pela via subaracnóidea.

Todas as pacientes receberam solução de Ringer com lactato $\left(10 \mathrm{ml} . \mathrm{kg}^{-1}\right)$, antes da realização da anestesia subaracnóidea. A punção subaracnóidea foi realizada com agulha de Quincke, calibre 25G, nos espaços intervertebrais $L_{2}-L_{3}$ ou $L_{3}-L_{4}$, com abordagem pela via mediana e com as pacientes em posição sentada.

Foi administrada a dose de $15 \mathrm{mg}$ de bupivacaína hiperbárica a $0,5 \%$ em 15 segundos, associada à morfina na dose de $0,1 \mathrm{mg}$ no grupo 1 e de $0,05 \mathrm{mg}$ no grupo 2 .

Ambos os grupos receberam cetoprofeno (100 mg) por via venosa no per-operatório, após o pinçamento do cordão umbilical e cetoprofeno por via oral a cada 8 horas no primeiro dia do pós operatório.

Um anestesiologista que não sabia em qual grupo estava incluída a paciente pesquisou a intensidade da dor e a presença de efeitos colaterais (sedação, prurido, náusea e vômito) no pós-operatório, 6,12 , e 24 horas após o término da cirurgia. A presença destes últimos também foi avaliada no per-operatório.
A intensidade da dor pós-operatória foi avaliada utilizando-se a escala numérica verbal, que variou de 0 a 10 , sendo 0 a ausência de dor e 10 a pior dor imaginável.

O grau de sedação foi avaliado por meio de escala numérica que variou de 0 a 3 , sendo 0 - acordada, sem evidências clínicas de sedação, 1 - sonolenta, respondendo a comandos verbais, 2 - sonolenta, não respondendo a comandos verbais, mas responsiva ao toque leve, e 3 - sedada intensamente, respondendo a estímulos dolorosos.

A intensidade do prurido foi avaliada pela escala numérica que variou de 0 a 4, sendo 0 - ausência de prurido, 1 - prurido leve, sem necessidade de coçar, 2 - prurido moderado, sendo necessário coçar ocasionalmente, 3 - prurido intenso com coceira constante e 4 - prurido intratável.

A presença e a intensidade da náusea foram avaliadas pela escala numérica verbal, que variou de 0 a 10, sendo 0 a ausência do sintoma e 10 a máxima náusea imaginável.

Também foram pesquisados a presença de vômito e o número de episódios ocorridos.

Aincidência de retenção urinária não foi estudada, porque as pacientes permanecem com cateter vesical nas primeiras 12 horas após a cesariana.

O método estatístico utilizado foi o teste $t$ de Student para idade, peso, altura, duração da cirurgia e anestesia, prova de Friedman para comparação de momentos em cada grupo, prova de Mann-Whitney para comparação dos grupos em cada momento e para o vômito, prova de Cochran e teste Exato de Fisher, sendo o valor de $p<0,05$ considerado significativo.

\section{RESULTADOS}

Os dois grupos foram homogêneos com relação à idade, ao peso, à altura, à duração da anestesia e da cirurgia (Tabela I).

Tabela I - Dados Antropométricos e Tempo de Duração da Cirurgia e Anestesia (Média \pm DP)

\begin{tabular}{lcc}
\hline & $\begin{array}{c}\mathrm{G} 1 \\
(\mathrm{n}=30)\end{array}$ & $\begin{array}{c}\mathrm{G} 2 \\
(\mathrm{n}=30)\end{array}$ \\
\hline Idade (anos) & $28 \pm 7$ & $25 \pm 7$ \\
Peso $(\mathrm{kg})$ & $76 \pm 14$ & $74 \pm 11$ \\
Altura (cm) & $159 \pm 8$ & $160 \pm 5$ \\
Duração da anestesia (min) & $110 \pm 29$ & $101 \pm 22$ \\
Duração da cirurgia (min) & $98 \pm 13$ & $89 \pm 11$ \\
\hline
\end{tabular}

Os dois grupos apresentaram comportamentos similares, em todos os momentos, com relação à intensidade da dor pós-operatória. Nas pacientes do grupo 1, o valor da mediana foi de 1 ; e no grupo 2 , foi de 2 na $24^{\text {a }}$ hora após o término da cirurgia (Tabela II).

Ambos os grupos comportaram-se de maneira idêntica em todos os momentos, com relação ao prurido, à sedação, à náusea e ao vômito (Tabela III).

Os valores referentes às medianas dos atributos estudados estão descritos nas tabelas IV (sedação), V (prurido), VI (náusea) e VII (vômito). 
Tabela II - Intensidade da Dor. Mediana dos Grupos em cada Momento. Estatísticas Calculadas para a Comparação dos Momentos dentro dos Grupos e dos Grupos em cada Momento

\begin{tabular}{lcccc}
\hline Dor & 6 horas & 12 horas & 24 horas & Comparação entre Momentos \\
\hline Grupo 1 & $0(0-0)$ & $0(0-0)$ & $1(0-1)$ & $6 \mathrm{~h} \leq 12 \mathrm{~h}$ \\
Grupo 2 & $0(0-2)$ & $0(0-2)$ & $2(0-5)$ & $(6 \mathrm{~h}=12 \mathrm{~h})<24 \mathrm{~h}$ \\
Comparação entre grupos & $\mathrm{G} 1=\mathrm{G} 2$ & $\mathrm{G} 1=\mathrm{G} 2$ & $\mathrm{G} 1=\mathrm{G} 2$ & \\
\hline
\end{tabular}

Tabela III - Prurido, Sedação, Náusea e Vômito no Per-Operatório e nas Primeiras 24 Horas após a Anestesia

\begin{tabular}{|c|c|c|c|c|c|c|c|c|c|}
\hline & $0,05 \mathrm{mg}$ & $0,1 \mathrm{mg}$ & $0,05 \mathrm{mg}$ & $0,1 \mathrm{mg}$ & $0,05 \mathrm{mg}$ & $0,1 \mathrm{mg}$ & $0,05 \mathrm{mg}$ & $0,1 \mathrm{mg}$ & \\
\hline & \multicolumn{2}{|c|}{ Per-Operatório } & \multicolumn{2}{|c|}{6 horas } & \multicolumn{2}{|c|}{12 horas } & \multicolumn{2}{|c|}{24 horas } & \\
\hline \multicolumn{10}{|l|}{ Prurido } \\
\hline Ausente & 25 & 24 & 14 & 10 & 20 & 22 & 27 & 28 & $p>0,05$ \\
\hline Leve & 0 & 2 & 8 & 9 & 3 & 4 & 1 & 0 & \\
\hline Moderado & 5 & 4 & 6 & 7 & 5 & 4 & 3 & 2 & \\
\hline Intenso & 0 & 0 & 2 & 4 & 2 & 0 & 0 & 0 & \\
\hline Intratável & 0 & 0 & 0 & 0 & 0 & 0 & 0 & 0 & \\
\hline \multicolumn{10}{|l|}{ Sedação } \\
\hline Acordada & 25 & 26 & 25 & 28 & 0 & 28 & 30 & 30 & \\
\hline Sonolenta, respondendo a comandos verbais & 5 & 4 & 5 & 2 & 0 & 2 & 0 & 0 & $p>0,05$ \\
\hline Sonolenta respondendo a toque & 0 & 0 & 0 & 0 & 0 & 0 & 0 & 0 & \\
\hline Sedação intensa & 0 & 0 & 0 & 0 & 0 & 0 & 0 & 0 & \\
\hline \multicolumn{10}{|l|}{ Náusea } \\
\hline Presente & 1 & 0 & 6 & 3 & 1 & 1 & 0 & 0 & $p>0,05$ \\
\hline Ausente & 29 & 30 & 24 & 27 & 29 & 29 & 0 & 0 & \\
\hline \multicolumn{10}{|l|}{ Vômito } \\
\hline Ausente & 30 & 30 & 27 & 28 & 30 & 30 & 30 & 30 & \\
\hline 1 a 2 episódios & 0 & 0 & 2 & 2 & & 0 & 0 & 0 & $p>0.05$ \\
\hline 3 a 4 episódios & 0 & 0 & 1 & 0 & 0 & 0 & 0 & 0 & \\
\hline$>4$ episódios & 0 & 0 & 0 & 0 & 0 & 0 & 0 & 0 & \\
\hline
\end{tabular}

Tabela IV - Intensidade da Sedação. Mediana dos Grupos em cada Momento. Estatísticas Calculadas para a Comparação dos Momentos dentro dos Grupos e dos Grupos em cada Momento

\begin{tabular}{lccccc}
\hline Sedação & 0 & 6 horas & 12 horas & 24 horas & Comparação entre Momentos \\
\hline Grupo 1 & 0 & 0 & 0 & 0 & $0 \mathrm{~h}=6 \mathrm{~h}=12 \mathrm{~h}=24 \mathrm{~h}$ \\
Grupo 2 & 0 & 0 & 0 & 0 & $0 \mathrm{~h}=6 \mathrm{~h}=12 \mathrm{~h}=24 \mathrm{~h}$ \\
Comparação entre grupos & $\mathrm{G} 1=\mathrm{G} 2$ & $\mathrm{G} 1=\mathrm{G} 2$ & $\mathrm{G} 1=\mathrm{G} 2$ & $\mathrm{G} 1=\mathrm{G} 2$ & \\
\hline
\end{tabular}

Tabela V - Intensidade do Prurido. Mediana dos Grupos em cada Momento. Estatísticas Calculadas para a Comparação dos Momentos dentro dos Grupos e dos Grupos em cada Momento

\begin{tabular}{|c|c|c|c|c|c|}
\hline Prurido & 0 & 6 horas & 12 horas & 24 horas & Comparação entre Momentos \\
\hline Grupo 1 & 0 & 1 & 0 & 0 & $6 h>(0 h=12 h=24 h)$ \\
\hline Grupo 2 & 0 & 1 & 0 & 0 & $6 h>(0 h=24 h)$ \\
\hline Comparação entre grupos & $\mathrm{G} 1=\mathrm{G} 2$ & $\mathrm{G} 1=\mathrm{G} 2$ & $\mathrm{G} 1=\mathrm{G} 2$ & $\mathrm{G} 1=\mathrm{G} 2$ & \\
\hline
\end{tabular}


Tabela VI - Náusea. Mediana dos Grupos em Cada Momento. Estatística Calculada para a Comparação dos Momentos Dentro de Cada Grupo e dos Grupos em cada Momento

\begin{tabular}{lccccc}
\hline Náusea & 0 & 6 horas & 12 horas & 24 horas & Comparação entre momentos \\
\hline Grupo 1 & 0 & 0 & 0 & 0 & $0 \mathrm{~h}=6 \mathrm{~h}=12 \mathrm{~h}=24 \mathrm{~h}$ \\
Grupo 2 & 0 & 0 & 0 & 0 & $0 \mathrm{~h}=6 \mathrm{~h}=12 \mathrm{~h}=24 \mathrm{~h}$ \\
Comparação entre grupos & $\mathrm{G} 1=\mathrm{G} 2$ & $\mathrm{G} 1=\mathrm{G} 2$ & $\mathrm{G} 1=\mathrm{G} 2$ & $\mathrm{G} 1=\mathrm{G} 2$ & \\
\hline
\end{tabular}

Tabela VII - Vômito, Número de Ocorrências em Cada Grupo nos Momentos. Estatísticas Calculadas para a Comparação dos Momentos dentro dos Grupos e dos Grupos em cada Momento

\begin{tabular}{lccccc}
\hline Vômito & 0 & 6 horas & 12 horas & 24 horas & Comparação entre momentos \\
\hline Grupo 1 & 0 & 3 & 0 & 0 & $6 \mathrm{~h}>(0 \mathrm{~h}=2 \mathrm{~h}=24 \mathrm{~h})$ \\
Grupo 2 & 0 & 2 & 0 & 0 & $6 \mathrm{~h}>(0 \mathrm{~h}=12 \mathrm{~h}=24 \mathrm{~h})$ \\
Comparação entre grupos & $\mathrm{G} 1=\mathrm{G} 2$ & $\mathrm{G} 1=\mathrm{G} 2$ & $\mathrm{G} 1=\mathrm{G} 2$ & $\mathrm{G} 1=\mathrm{G} 2$ & \\
\hline
\end{tabular}

\section{DISCUSSÃO}

Os resultados deste estudo mostraram que a morfina, nas doses de 0,05 ou $0,1 \mathrm{mg}$ administradas no espaço subaracnóideo, e o cetoprofeno porvia venosa e oral foram eficientes e agiram de maneira similar ao proporcionar analgesia pós-operatória em pacientes submetidas à cesariana.

Está descrito que pequenas doses de opióides de longa duração, utilizadas pela via subaracnóidea, impedem a ocorrência da dor pós-operatória do parto cesariano e que 0,01 mg de morfina proporciona analgesia pós-operatória de mesma qualidade que as doses mais elevadas e com menor incidência de efeitos colaterais ${ }^{8,9}$.

Análise de resultados obtida por meio de estudo de meta-análise mostrou que $0,1 \mathrm{mg}$ de morfina introduzido no espaço subaracnóideo é a dose adequada para analgesia após cesariana ${ }^{7}$. Entretanto, alguns autores constataram que esta dose foi eficaz para bloqueio da dor apenas quando associada a outros analgésicos ${ }^{10}$.

Tem sido ressaltada a importância da abordagem multimodal para controle da dor, com combinações de drogas que atuam por meio de mecanismos diferentes.

A combinação de opióides com antiinflamatórios não esteróides (AINE) resulta em ação sinérgica, o que proporciona controle satisfatório da dor pós-operatória ${ }^{11}$. Foi observado que quando se utilizam os AINE, reduz-se a necessidade de outros analgésicos para prover analgesia adequada ${ }^{11,12}$.

A morfina, na dose de 0,025 mg por via subaracnóidea, associada aos antiinflamatórios não esteróides (diclofenaco), proporcionou boa qualidade de analgesia, com mínimos efeitos colaterais ${ }^{13}$

Enquanto o principal local de ação da morfina subaracnóidea é o receptor opióide na substância gelatinosa do corno dorsal da medula espinhal ${ }^{14}$, o cetoprofeno possui ação inibitória das prostaglandinas centrais e periféricas, atuando na síntese e atividade de outras substâncias neuroativas que desempenham papel na instalação do estímulo nociceptivo no corno posterior da medula.
Ador após cesariana possui três componentes - o derivado da incisão cirúrgica, o secundário à separação do reto, a dor muscular profunda e o proveniente das contrações uterinas ${ }^{15}$.

O efeito inibidor da síntese das prostaglandinas do cetoprofeno pode ter contribuído para o alívio da dor secundária às contrações uterinas, melhorando, desta forma, a qualidade da analgesia pós-operatória. O efeito antiinflamatório dos AINE alivia a dor da incisão cirúrgica ${ }^{1,6}$.

No presente estudo, as doses de morfina de 0,05 mg e 0,1 mg associadas ao cetoprofeno foram consideradas eficazes em bloquear a dor nas primeiras 12 horas de pós-operatório. Após 24 horas de cirurgia a dor foi de intensidade leve.

Estes resultados estão em concordância com aqueles observados na meta-análise, que mostraram que $0,1 \mathrm{mg}$ de morfina subaracnóidea resulta em pelo menos 11 horas de analgesia eficaz ${ }^{7}$.

Quanto aos efeitos colaterais, o prurido é o mais freqüente. Os resultados obtidos pelo estudo de meta-análise mostraram incidência de $43 \%$.

Não há, na literatura, consenso se a ocorrência de prurido é dose-dependente ${ }^{8,17-19}$ ou não tem relação com a dose ${ }^{9}$. Nesta pesquisa, a ocorrência de prurido foi de $66 \%$, não foi dose-dependente e apareceu principalmente na $6^{a}$ hora após a cirurgia. Alguns autores observaram que o prurido acontece, com maior freqüência, entre a $6^{a}$ e $9^{a}$ horas após o término da cirurgia ${ }^{19}$.

Em pacientes obstétricas, a incidência de prurido após administração de morfina, tanto no espaço peridural como no subaracnóideo, é freqüente e pode limitar seu uso ${ }^{20}$. Acredita-se que, neste grupo de pacientes, exista maior vulnerabilidade ao prurido em decorrência de ligação alterada do opióide com seu local receptor, o que pode ser causada pela competição com o estrógeno ${ }^{21}$.

A maior facilidade para que haja dispersão cefálica das drogas administradas pela via espinhal em gestantes de termo também contribuiria para a maior incidência de prurido neste grupo de pacientes ${ }^{22}$. 
O mecanismo que desencadeia o prurido após administração neuroaxial de opióide não é completamente compreendido. Parece não estar relacionado à liberação de histamina, visto que bloqueadores de histamina são ineficazes para tratá-lo ${ }^{23}$.

A ativação dos receptores opióides $\mu$ é responsável pela modulação da dor e de outros efeitos colaterais, como o prurido ${ }^{21}$.

Os opióides também estimulam a zona quimiorreceptora de gatilho na área postrema, na porção caudal do quarto ventrículo, desencadeando náusea.

Com relação à emese, resultados referentes ao estudo de meta-análise revelaram que a incidência de náusea foi de $10 \%$ e de vômito, $12 \%$, para doses de $0,1 \mathrm{mg}$ de morfina pela via subaracnóidea ${ }^{7}$.

Na presente pesquisa, observou-se que $20 \%$ das pacientes que receberam 0,05 de morfina e $10 \%$ daquelas que receberam $0,1 \mathrm{mg}$ apresentaram náusea. Já, com relação ao vômito, aproximadamente $10 \%$ das pacientes apresentaram o efeito colateral. Estes resultados estão de acordo com os observados na literatura ${ }^{7}$. Entretanto, diferentemente da maioria dos estudos, nos quais a maior incidência de emese aconteceu com doses mais elevadas de morfina ${ }^{9,13,18,24}$, o constatado, neste estudo, foi a ocorrência similar de náusea e vômito em ambos os grupos.

Os resultados obtidos nesta pesquisa mostram que a morfina, nas doses de $0,05 \mathrm{mg}$ ou $0,1 \mathrm{mg}$ administradas no espaço subaracnóideo, associada ao cetoprofeno pela via venosa e oral, apresentou a mesma qualidade de analgesia após parto cesariano e a mesma ocorrência de efeitos colaterais.

\section{Effects of Low Spinal Morphine Doses Associated to Intravenous and Oral Ketoprofen in Patients Submitted to Cesarean Sections}

Eliana Marisa Ganem, TSA, M.D.; Norma Sueli Pinheiro Módolo, TSA, M.D.; Fábio Ferrari, M.D.; Francisco Carlos Obata Cordon,M.D.; Edgar Shiguero Koguti, M.D.; Yara Marcondes Machado Castiglia, TSA, M.D.

\section{INTRODUCTION}

Spinal anesthesia is commonly used for Cesarean sections. It is easy to perform and shows fast onset, also inducing excellent muscle relaxation and intense analgesia, thus offering optimal perioperative conditions ${ }^{1,2}$

Since the discovery of spinal cord opioid receptors during the 70 's, clinical application of opioids, both in the epidural and the spinal space, has revolutionized obstetric analgesia ${ }^{3}$. Fentanyl, sufentanil and morphine are opioids which may be added to local anesthetics to improve perioperative analge- $\mathrm{sia}^{4}$, being morphine the drug of choice for postoperative analgesia ${ }^{5}$.

Opioids, however, may trigger some undesirable side-effects, such as nausea, vomiting, pruritus, respiratory depression, urinary retention and sedation, the intensity of which may be dose-dependent ${ }^{6}$.

Recommended spinal morphine dose is $0.1 \mathrm{mg}^{7,8}$, but there are clinical trials showing that morphine could reach its peak effect with doses below $0.1 \mathrm{mg}^{5}$. It is believed that lower spinal morphine doses, associated to other analgesic drugs, could be effective in decreasing postoperative pain with a lower incidence of side-effects.

This study aimed at evaluating postoperative analgesia and the incidence of side-effects in patients submitted to Cesarean sections under spinal anesthesia with bupivacaine and $0.05 \mathrm{mg}$ and $0.1 \mathrm{mg}$ morphine associated to intravenous and oral ketoprofen.

\section{METHODS}

After the Clinical Research Ethics Committee approval and with patients written consent, 60 term pregnant women, physical status ASA I or II, undergoing elective Cesarean sections with term and non-suffering fetuses were included in the study. Patients were randomly distributed in two groups according to the morphine dose added to local spinal anesthetics.

All patients were given $10 \mathrm{ml} . \mathrm{kg}^{-1}$ lactated Ringer's before spinal anesthesia. Puncture was performed with $25 \mathrm{G}$ Quincke needles in $L_{2}-L_{3}$ or $L_{3}-L_{4}$ interspace through the median approach and with patients in the sitting position.

Patients received $15 \mathrm{mg}$ of $0.5 \%$ hyperbaric bupivacaine in 15 seconds associated to $0.1 \mathrm{mg}$ morphine in group 1 and $0.05 \mathrm{mg}$ morphine in group 2 .

Both groups were given $100 \mathrm{mg}$ intravenous ketoprofen in the perioperative period, after umbilical cord clamping, and oral ketoprofen at 8-hour intervals in the first postoperative day. Postoperative pain and the incidence of side-effects (sedation, pruritus, nausea and vomiting) 6, 12 and 24 hours after surgery, were evaluated by an anesthesiologist blind to patients group. Side-effects were also evaluated in the perioperative period.

Postoperative pain intensity was evaluated by a 0 to 10 verbal numerical scale, where 0 was no pain and 10 the worst possible pain.

Sedation was evaluated by a 0 to 3 numerical scale, being 0 awaken with no clinical evidences of sedation, 1 - sleepy, responding to verbal commands, 2 - sleepy, not responding to verbal commands but responsive to mild touch, and 3 deeply sedated, responding to painful stimulation.

Pruritus was evaluated by a 0 to 4 numerical scale, where 0 no pruritus, 1 - mild pruritus with no itching, 2 - moderate pruritus needing occasional itching, 3 - severe pruritus with constant itching, and 4 - untreatable pruritus.

Nausea also was evaluated by a 0 to 10 verbal numerical scale, where 0 was no symptom and 10 the worst imaginable nausea. 
The incidence and number of vomiting episodes were also registered.

Urinary retention was not evaluated because patients remained with vesical catheter during the first 12 postoperative hours.

Student's $t$ test was used for statistical analysis of age, weight, height, surgery and anesthesia duration. Friedman's test was used to compare moments within each group, Mann-Whitney test was used to compare groups in each moment, and Cochran and Fisher Exact tests were used for vomiting, being significant $p<0.05$.

\section{RESULTS}

Groups were homogeneous in age, weight, height and surgery and anesthesia duration (Table I).
Table I - Demographics Data, Surgery and Anesthesia Duration (Mean \pm SD)

\begin{tabular}{lcc}
\hline & $\begin{array}{c}\mathrm{G} 1 \\
(\mathrm{n}=30)\end{array}$ & $\begin{array}{c}\mathrm{G} 2 \\
(\mathrm{n}=30)\end{array}$ \\
\hline Age (years) & $28 \pm 7$ & $25 \pm 7$ \\
Weight $(\mathrm{kg})$ & $76 \pm 14$ & $74 \pm 11$ \\
Height $(\mathrm{cm})$ & $159 \pm 8$ & $160 \pm 5$ \\
Anesthesia duration (min) & $110 \pm 29$ & $101 \pm 22$ \\
Surgery duration (min) & $98 \pm 13$ & $89 \pm 11$ \\
\hline
\end{tabular}

Groups were also similar in all moments as for postoperative pain intensity. Median for group 1 was 1 and for group 2 was 2 in the $24^{\text {th }}$ postoperative hour (Table II).

Groups were identical in all moments regarding the incidence of pruritus, sedation, nausea and vomiting (Table III).

Medians of studied attributes are shown in tables IV (sedation), V (pruritus), VI (nausea) and VII (vomiting).

Table II - Pain Intensity. Groups Median in each Moment. Statistical Analysis to Compare Moments within Groups and Groups within each Moment

\begin{tabular}{lcccc}
\hline Pain & 6 hours & 12 hours & 24 hours & Comparison among Moments \\
\hline Group 1 & $0(0-0)$ & $0(0-0)$ & $1(0-1)$ & $6 \mathrm{~h} \leq 12 \mathrm{~h}$ \\
Group 2 & $0(0-2)$ & $0(0-2)$ & $2(0-5)$ & $(6 \mathrm{~h}=12 \mathrm{~h})<24 \mathrm{~h}$ \\
Comparison between groups & $\mathrm{G} 1=\mathrm{G} 2$ & $\mathrm{G} 1=\mathrm{G} 2$ & $\mathrm{G} 1=\mathrm{G} 2$ & \\
\hline
\end{tabular}

Table III - Pruritus, Sedation, Nausea and Vomiting in the Perioperative Period and the first 24 Post-Anesthetic Hours

\begin{tabular}{|c|c|c|c|c|c|c|c|c|c|}
\hline & $0.05 \mathrm{mg}$ & $0.1 \mathrm{mg}$ & $0.05 \mathrm{mg}$ & $0.1 \mathrm{mg}$ & $0.05 \mathrm{mg}$ & $0.1 \mathrm{mg}$ & $0.05 \mathrm{mg}$ & $0.1 \mathrm{mg}$ & \\
\hline & \multicolumn{2}{|c|}{ Perioperative Period } & \multicolumn{2}{|c|}{6 hours } & \multicolumn{2}{|c|}{12 hours } & \multicolumn{2}{|c|}{24 hours } & \\
\hline \multicolumn{10}{|l|}{ Pruritus } \\
\hline Absent & 25 & 24 & 14 & 10 & 20 & 22 & 27 & 28 & $p>0.05$ \\
\hline Mild & 0 & 2 & 8 & 9 & 3 & 4 & 1 & 0 & \\
\hline Moderate & 5 & 4 & 6 & 7 & 5 & 4 & 3 & 2 & \\
\hline Severe & 0 & 0 & 2 & 4 & 2 & 0 & 0 & 0 & \\
\hline Untreatable & 0 & 0 & 0 & 0 & 0 & 0 & 0 & 0 & \\
\hline \multicolumn{10}{|l|}{ Sedation } \\
\hline Awaken & 25 & 26 & 25 & 28 & 0 & 28 & 30 & 30 & \\
\hline Sleepy, responding to verbal commands & 5 & 4 & 5 & 2 & 0 & 2 & 0 & 0 & $p>0.05$ \\
\hline Sleepy responding to touch & 0 & 0 & 0 & 0 & 0 & 0 & 0 & 0 & \\
\hline Deep sedation & 0 & 0 & 0 & 0 & 0 & 0 & 0 & 0 & \\
\hline \multicolumn{10}{|l|}{ Nausea } \\
\hline Present & 1 & 0 & 6 & 3 & 1 & 1 & 0 & 0 & $p>0.05$ \\
\hline Absent & 29 & 30 & 24 & 27 & 29 & 29 & 0 & 0 & \\
\hline \multicolumn{10}{|l|}{ Vomiting } \\
\hline Absent & 30 & 30 & 27 & 28 & 30 & 30 & 30 & 30 & \\
\hline 1 to 2 episodes & 0 & 0 & 2 & 2 & & 0 & 0 & 0 & $p>0.05$ \\
\hline 3 to 4 episodes & 0 & 0 & 1 & 0 & 0 & 0 & 0 & 0 & \\
\hline$>4$ episodes & 0 & 0 & 0 & 0 & 0 & 0 & 0 & 0 & \\
\hline
\end{tabular}


Table IV - Sedation Intensity. Groups Median in each Moment. Statistical Analysis to Compare Moments within Groups and Groups in each Moment

\begin{tabular}{lccccc}
\hline Sedation & 0 & 6 hours & 12 hours & 24 hours & Comparison among moments \\
\hline Group 1 & 0 & 0 & 0 & 0 & $0 \mathrm{~h}=6 \mathrm{~h}=12 \mathrm{~h}=24 \mathrm{~h}$ \\
Group 2 & 0 & 0 & 0 & 0 & $0 \mathrm{~h}=6 \mathrm{~h}=12 \mathrm{~h}=24 \mathrm{~h}$ \\
Comparison between groups & $\mathrm{G} 1=\mathrm{G} 2$ & $\mathrm{G} 1=\mathrm{G} 2$ & $\mathrm{G} 1=\mathrm{G} 2$ & $\mathrm{G} 1=\mathrm{G} 2$ & \\
\hline
\end{tabular}

Table V - Pruritus Intensity. Groups Median in each Moment. Statistical Analysis to Compare Moments within Groups and Groups in each Moment

\begin{tabular}{lccccc}
\hline Pruritus & 0 & 6 hours & 12 hours & 24 hours & Comparison among Moments \\
\hline Group 1 & 0 & 1 & 0 & 0 & $6 \mathrm{~h}>(0 \mathrm{~h}=12 \mathrm{~h}=24 \mathrm{~h})$ \\
Group 2 & 0 & 1 & 0 & 0 & $6 \mathrm{~h}>(0 \mathrm{~h}=24 \mathrm{~h})$ \\
Comparison between groups & $\mathrm{G} 1=\mathrm{G} 2$ & $\mathrm{G} 1=\mathrm{G} 2$ & $\mathrm{G} 1=\mathrm{G} 2$ & $\mathrm{G} 1=\mathrm{G} 2$ & \\
\hline
\end{tabular}

Table VI - Nausea. Groups Median in each Moment. Statistical Analysis to Compare Moments within Groups and Groups in each Moment

\begin{tabular}{lccccc}
\hline Nausea & 0 & 6 hours & 12 hours & 24 hours & Comparison among Moments \\
\hline Group 1 & 0 & 0 & 0 & 0 & $0 \mathrm{~h}=6 \mathrm{~h}=12 \mathrm{~h}=24 \mathrm{~h}$ \\
Group 2 & 0 & 0 & 0 & 0 & $0 \mathrm{~h}=6 \mathrm{~h}=12 \mathrm{~h}=24 \mathrm{~h}$ \\
Comparison between groups & $\mathrm{G} 1=\mathrm{G} 2$ & $\mathrm{G} 1=\mathrm{G} 2$ & $\mathrm{G} 1=\mathrm{G} 2$ & $\mathrm{G} 1=\mathrm{G} 2$ & \\
\hline
\end{tabular}

Table VII - Vomiting. Groups Median in each Moment. Statistical Analysis to Compare Moments within Groups and Groups in each Moment

\begin{tabular}{lccccc}
\hline Vomiting & 0 & 6 hours & 12 hours & 24 hours & Comparison among Moments \\
\hline Group 1 & 0 & 3 & 0 & 0 & $6 \mathrm{~h}>(0 \mathrm{~h}=2 \mathrm{~h}=24 \mathrm{~h})$ \\
Group 2 & 0 & 2 & 0 & 0 & $6 \mathrm{~h}>(0 \mathrm{~h}=12 \mathrm{~h}=24 \mathrm{~h})$ \\
Comparison between groups & $\mathrm{G} 1=\mathrm{G} 2$ & $\mathrm{G} 1=\mathrm{G} 2$ & $\mathrm{G} 1=\mathrm{G} 2$ & $\mathrm{G} 1=\mathrm{G} 2$ & \\
\hline
\end{tabular}

\section{DISCUSSION}

This study has shown that $0.05 \mathrm{mg}$ or $0.1 \mathrm{mg}$ spinal morphine and intravenous and oral ketoprofen were effective with similar postoperative analgesia in patients submitted to Cesarean sections. It has been described that low long-lasting spinal opioid doses prevent postoperative pain of Cesarean deliveries and that $0.01 \mathrm{mg}$ morphine provides postoperative analgesia similar to higher doses, with less side-effects ${ }^{8,9}$.

A meta-analysis study has shown that $0.1 \mathrm{mg}$ spinal morphine is adequate for post-Cesarean section analgesia ${ }^{7}$. Some authors, however, have observed that this dose was effective for pain relief only when associated to other analge$\operatorname{sics}^{10}$.

It has been highlighted the importance of a multimodal approach for pain control, with combination of drugs acting through different mechanisms.

The combination of opioids with non-steroid anti-inflammatory drugs (NSAIDs) results in a synergistic action promoting satisfactory postoperative pain control ${ }^{11}$. It has been observed that NSAIDs decrease the need for other analgesics to promote adequate analgesia ${ }^{11,12}$.
Spinal $0.025 \mathrm{mg}$ morphine associated to NSAIDs (diclofenac) has promoted good quality analgesia with minor side-effects ${ }^{13}$.

While spinal morphine primary action site is the opioid receptor in the gelatinous substance of the spinal cord dorsal horn ${ }^{14}$, ketoprofen inhibits central and peripheral prostaglandins, acting in the synthesis and activity of other neuroactive substances which play important roles in nociceptive stimulation in the spinal cord posterior horn.

Post-Cesarean pain features three components: one derived from surgical incision, another secondary to retus separation and deep muscle pain and the las one, derived from uterine contractions $^{15}$.

Ketoprofen prostaglandin synthesis inhibition may have contributed to uterine contractions pain relief, thus improving postoperative analgesia. NSAIDs anti-inflammatory effects relieve surgical incision pain ${ }^{1,6}$.

In our study, $0.05 \mathrm{mg}$ and $0.1 \mathrm{mg}$ morphine doses associated to ketoprofen were considered effective in blocking pain during the first 12 postoperative hours. There has been mild pain after 24 hours. 
These results are in line with those observed in the meta-analysis, which have shown that $0.1 \mathrm{mg}$ spinal morphine results in at least 11 hours of effective analgesia ${ }^{7}$. Pruritus is the most frequent side-effect. Meta-analysis results have shown an incidence of $43 \%$.

There is no consensus in the literature whether pruritus is dose-dependent ${ }^{8,17-19}$ or not $^{9}$. In our study, the incidence of pruritus was $66 \%$, not dose-dependent and appeared mainly in the $6^{\text {th }}$ postoperative hour. Some authors have observed that pruritus is more frequent between the $6^{\text {th }}$ and $9^{\text {th }}$ postoperative hour ${ }^{19}$. In obstetric patients, the incidence of pruritus after epidural or spinal morphine administration is frequent and may limit its use ${ }^{20}$. It is believed that this group of patients is more vulnerable to pruritus due to a change in opioid binding to its receptor site, which could be caused by estrogen competition ${ }^{21}$.

The facilitation of spinal drugs cephalad spread in term pregnant women would also contribute to a higher incidence of pruritus ${ }^{22}$.

Pruritus triggering mechanism after neuraxial opioid administration is not totally understood. It seems to be unrelated to histamine release, since histamine blockers are ineffective to treat it ${ }^{23}$.

The activation of $\mu$ opioid receptors is responsible for pain modulation and other side-effects, such as pruritus ${ }^{21}$.

Opioids also stimulate chemoreceptor triggering zone in the postreme area in the caudal portion of the fourth ventricle, inducing nausea.

Meta-analysis results showed $10 \%$ of nausea and $12 \%$ of vomiting for $0.1 \mathrm{mg}$ spinal morphine ${ }^{7}$.

Our study has shown that $20 \%$ of patients receiving $0.05 \mathrm{mg}$ morphine and $10 \%$ of those receiving $0.1 \mathrm{mg}$ had nausea. Approximately $10 \%$ of patients had vomiting as side-effect. These results are in line with the literature ${ }^{7}$. However, differently from most studies where the higher incidence of emesis was related to higher morphine doses ${ }^{9,13,18,24}$, our study has observed that there has been a similar incidence of nausea and vomiting in both groups.

Our results have shown that $0.05 \mathrm{mg}$ or $0.1 \mathrm{mg}$ spinal morphine associated to intravenous and oral ketoprofen have provided the same post-Cesarean section analgesia with the same incidence of side-effects.

\section{REFERÊNCIAS - REFERENCES}

01. Schnider SM, Levinson G - Anesthesia for Cesarean Section, em: Schnider SM, Levinson G, Rosen AM - Anesthesia for Obstetrics. Baltimore; Williams \& Wilkins, 1993;211-239.

02. Bhavani-Shankar - Anesthesia for cesarean delivery. Probl in Anesth, 1999;3:333-343

03. Cohe S - Strategies for labor pain relief - past, present and future. Acta Anaesthesiol Scand, 1997;17-21.

04. Palmer CM, Voulgaropoulos D, Alvens D - Subarachnoid fentanyl augments lidocaine spinal anesthesia for cesarean section. Reg Anesth, 1995;20:389-394.
05. Sibilla C, Albertazzi P, Zateli R et al - Pain relief after caesarean section: comparison of different techniques of morphine administration. Int J Obstet Anaesth, 1994;3:203-207.

06. Stoelting RK - Opioid Agonists and Antagonists, em: Stoelting RK - Pharmacology and Physiology in Anesthetic Practice. Philadelphia: Lippincott-Raven Publishers, 1999;77-112.

07. Dahl JB, Jeppesen IS, Jorgensen $\mathrm{H}$ et al - Intraoperative and postoperative analgesic efficacy and adverse effects of intrathecal opioids in patients undergoing cesarean section with spinal anesthesia. Anesthesiology, 1999; 91:1919-1927.

08. Palmer CM, Emerson S, Volgoropolous D et al - Dose-response relationship of intrathecal morphine for post-cesarean analgesia. Anesthesiology, 1999;90:437-444.

09. Milner AR, Bogod DG, Harwood RJ et al - Intrathecal administration of morphine for elective caesarean section. A comparison between $0.1 \mathrm{mg}$ and 0.2 mg. Anaesthesia, 1996;51:871-873.

10. Swart M, Swell J, Thomas D - Intrathecal morphine for caesarean section: an assessment of pain relief, satisfaction an side-effects. Anaesthesia, 1997;52:364-381.

11. Souter AJ, Fredman B, White PF - Controversies in the preoperative use of nonsteroidal anti-inflammatory drugs. Anesth Analg, 1994;79:1178-1190.

12. Luthman J, Kay NH, White JB - The morphine sparing effects of diclofenac sodium following cesarean section under spinal anesthesia. Int J Obstet Anesth, 1994;3:82-86.

13. Cardoso MMSC, Carvalho JCA, Amaro AR et al - Small doses of intrathecal morphine combined with systemic diclofenac for postoperative pain control after cesarean delivery. Anesth Analg, 1998;86:538-541.

14. Yaksh TL, Wilson PR, Kaikorf et al - Analgesia produced by a spinal action of morphine and effects upon parturition in the rat. Anesthesiology, 1979;51:386-392.

15. Pavy TJ, Gambling DR, Merrick PM et al - Rectal indomethacin potentiates morphine analgesia after caesarean delivery. Anaesth Int Care, 1995;23:555-559.

16. Cohen SE, Desai JB, Ratner EF et al - Ketorolac and spinal morphine for postcesarean analgesia. Int J Obstet Anesth, 1996;5: 14-18.

17. Chadwick HS, Ready LB - Intrathecal and epidural morphine sulfate for postcesarean analgesia a clinical comparison. Anesthesiology, 1988;68:925-929.

18. Uchiyama A, Ueyama $\mathrm{H}$, Nakano S et al - Low dose intrathecal morphine and pain relief following caesarean section. Int J Obst Anesth, 1994;3:87-91.

19. Slappendel R, Weber EWG, Benraad B et al - Itching after intrathecal morphine. Incidence and treatment. Eur J Anaesth, 2000;17:616-621.

20. Fuller JG, McMorland GH, Douglas MJ et al - Epidural morphine for analgesia after caesarean section: a report of 4880 patients. Can J Anaesth, 1990;37:636-640

21. Bromage $P R$ - The price of intraspinal narcotic analgesia: basic constraints. Anesth Analg, 1981;60:461-463.

22. Warwick JP, Keearns CF, Scott WE - The effect of subhypnotic doses of propofol on the incidence of pruritus after intrathecal morphine for caesarean section - Anaesthesia, 1997;52: 265-275.

23. Krause L, Shuster S Mechanism of action of antipruritic drugs. BMJ, 1983;287:1199-1200.

24. Yang T, Breen TW, Archer D et al - Comparison of $0.25 \mathrm{mg}$ and $0.1 \mathrm{mg}$ intrathecal morphine for analgesia after cesarean and section. Can J Anaesth, 1999;46:856-860. 


\section{RESUMEN}

Ganem EM, Módolo NSP, Ferrari F, Cordon FCO, Koguti ES, Castiglia YMM - Efectos de la Asociación entre Pequeñas Dosis Subaracnóideas de Morfina y Cetoprofeno Venoso y Oral en Pacientes Sometidas a Cesariana

JUSTIFICATIVA Y OBJETIVOS: Pequeñas dosis subaracnóideas de morfina son eficaces en reducir el dolor pos-operatorio de pacientes sometidas a cesariana, con menor incidencia de efectos colaterales. El objetivo de esta pesquisa fue evaluar la calidad de la analgesia pos-operatoria y la ocurrencia de efectos colaterales en pacientes sometidas a cesariana, bajo anestesia subaracnóidea con bupivacaína hiperbárica y morfina en las dosis de 0,05 mg y 0,1 mg, asociadas al cetoprofeno por las vías venosa y oral.

MÉTODO: Participaron del estudio 60 gestantes de término, estado físico ASA I y II, que fueron sometidas a cesariana electiva. Las pacientes fueron divididas en dos grupos: grupo 1 - morfina 0,1 mg, grupo 2 - 0,05 mg, asociada a $15 \mathrm{mg}$ de bupivacaína hiperbárica. Todas recibieron cetoprofeno (100 mg) por vía venosa en el per-operatorio y por vía oral a cada 8 horas en el primer día del pos-operatorio. Las pacientes fueron evaluadas 6, 12 y 24 horas después del término de la cirugía, con relación a la intensidad del dolor y presencia de efectos colaterales (sedación, prurito, náusea y vómito). La presencia de estos últimos también fue evaluada en el per-operatorio.

RESULTADOS: Ambos grupos fueron idénticos cuanto a los datos antropométricos y la duración de la cirugía y de la anestesia. También fueron homogéneos con relación a la intensidad del dolor pos-operatorio y a la presencia de prurito, sedación, náusea y vómito.

CONCLUSIONES: La morfina, en las dosis de 0,05 mg y 0,1 mg administradas en el espacio subaracnóideo, asociada al cetoprofeno por las vías venosa y oral, presentó la misma calidad de analgesia pos-operatoria y determinó la misma ocurrencia de efectos colaterales. 